\title{
Non-unique solution for combined-convection assisting flow over vertical flat plate
}

\author{
K VENKATASUBBAIAH, AMRITA MITTAL and T K SENGUPTA* \\ Department of Aerospace Engineering, Indian Institute of Technology, \\ Kanpur 208 016, India \\ *e-mail: tksen@iitk.ac.in \\ MS received 5 December 2005; revised 6 July 2006
}

\begin{abstract}
Non-unique solutions of flow and temperature field are reported here for the first time for non-similar flows given by the laminar boundary layer equations for combined-convection flow past a vertical flat plate. The solution of the boundary layer equation for natural convection constitutes the self-similar solution whose perturbation with respect to the small parameter $(\varepsilon)$, which is inversely proportional to the square root of the Richardson number $\left(G_{x}\right)$, provides the nonsimilar solution. Solutions obtained by the shooting method indicate two sets for the self-similar solution $(\varepsilon=0)$ - one of them showing positive velocity everywhere inside the shear layer (well-known oft-reported physical result). The other self-similar solution shows that recirculation in the outer part of the shear layer may not be physical - as it has not been experimentally demonstrated so far. In contrast, the perturbative part of the non-similar solution $(\varepsilon \neq 0)$ is seen to be either convergent or divergent depending upon the choice of integration domain of the shear layer equations - bringing forth the question of the validity of such perturbation procedures and possible stability of the basic solution itself.
\end{abstract}

Keywords. Combined-convection flow; assisting flow; non-unique solution; non-similar solution.

\section{Introduction}

A two-dimensional boundary layer developing over a heated vertical flat plate is an example of free-convection flow. A buoyancy force is created by density changes due to temperature variation in the flow. In the present work, we are interested in studying the small modified effects of forced convection on the natural convection boundary layer. In such cases, the flow about the plate is a perturbation of free convection by forced convection in assisting or opposing the buoyancy force, as indicated in figure 1 . In case of a heated plate, the buoyancy force assists mean convection, if the latter is directed from bottom to top and it opposes mean convection when the latter is from top to bottom.

There are references that report the numerical solution of the combined convection problem over horizontal and vertical plates. While Schneider (1979) provided the similarity solution 

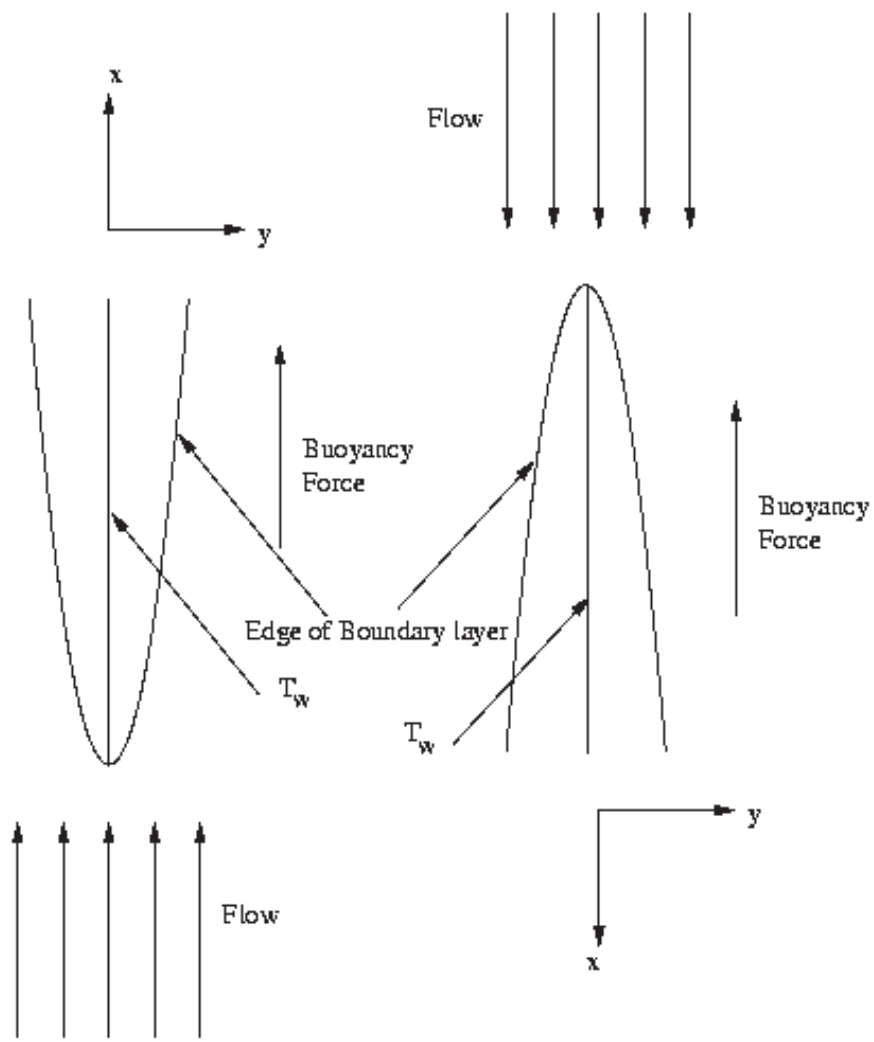

a Assisting Elow

b. Opposing Elow

Figure 1. Mixed convection flow over a vertical flat plate.

past a horizontal cooled plate, in later work Schneider \& Wasel (1985) reported finding a singularity with a finite wall shear stress. Steinrück (1994) related this problem with unboundedness of the evolution operator of the linearized equations. It was shown that near the leading edge of the plate, an asymptotic expansion of the solution is not unique. In contrast, for the boundary layer flow above a heated vertical or an inclined heated or cooled plate no such difficulties have been reported so far. In this paper, we investigate the case of a vertical heated plate for the assisting flow case shown in figure 1, to show the existence of non-unique solutions and possible breakdown of the asymptotic expansion of such combined convection flows.

Here, we are interested in obtaining the solution of the governing equation in a region where the forced convection effects on free convection are small. It is well known (as discussed in Cebeci \& Bradshaw (1984) and Oosthuizen \& Naylor (1999)) that such flows do not yield self-similar solutions and the small departure from selfsimilarity can be expressed by using a perturbative technique under the boundary layer and Boussinesq assumption. This can be assessed in the manner given in the next section. 


\section{Governing equations}

With the boundary layer approximations, the governing equations are expressed in terms of the conservation equations for mass, momentum and energy, which are given respectively by (for more details see Ooshtuizen \& Naylor 1999),

$$
\begin{aligned}
\frac{\partial u}{\partial x}+\frac{\partial v}{\partial y} & =0, \\
u \frac{\partial u}{\partial v}+v \frac{\partial u}{\partial y} & =v \frac{\partial^{2} u}{\partial y^{2}} \pm \beta g\left(T-T_{\infty}\right), \\
u \frac{\partial T}{\partial x}+v \frac{\partial T}{\partial y} & =\frac{k}{\rho C_{p}} \frac{\partial^{2} T}{\partial y^{2}} .
\end{aligned}
$$

The $(+)$ and $(-)$ signs on the buoyancy term in the momentum conservation equation refer to assisting and opposing flows respectively. For the present study, we consider the case when the buoyancy assists the flow and when the natural convection dominates over the forced convection. The physical boundary conditions for solving (1)-(3) is given later following (10).

Irrespective of the fact whether a similarity solution exists or not, one can introduce the similarity variable used in free convection (for which similarity solution exists) as given by,

$$
\eta=\frac{y}{x} \mathrm{Gr}_{x}^{1 / 4}=y\left[\frac{\beta g\left(T_{w}-T_{\infty}\right)}{v^{2} x}\right]^{1 / 4},
$$

where $\mathrm{Gr}_{x}$ is the local Grashof number.

One can define the non-similar velocity and temperature profiles by non-dimensional velocity as,

$$
u /\left[\beta g\left(T_{w}-T_{\infty}\right) x\right]^{1 / 2}=F^{\prime}(\eta, x)
$$

where $\left[\beta g\left(T_{w}-T_{\infty}\right) x\right]^{1 / 2}$ is the reference velocity that is used for the free convection problem and the prime indicates differentiation with respect to $\eta$. The non-dimensional temperature field is similarly defined by,

$$
\left(T-T_{\infty}\right) /\left(T_{w}-T_{\infty}\right)=H(\eta, x),
$$

where $T_{w}$ and $T_{\infty}$ are the temperatures at the wall of the vertical plate and the free stream respectively. Substituting (4)-(6) in (2) and (3), we get the boundary layer equations as,

$$
\begin{aligned}
F^{\prime \prime \prime}+\left(3 F F^{\prime \prime} / 4\right)-\left(F^{\prime 2} / 2\right)+H & =0, \\
H^{\prime \prime}+(3 / 4) \operatorname{Pr} F H^{\prime} & =0 .
\end{aligned}
$$

Although these equations suggest the possibility of obtaining self-similar solutions, as there are no explicit $x$ dependent terms, the boundary condition at large $\eta$ indicates dependence on $G_{x}^{-1 / 2}$. For large $\eta: F^{\prime} \rightarrow u_{\infty} /\left[\beta g\left(T_{w}-T_{\infty}\right) x\right]^{1 / 2}=\operatorname{Re}_{x} / \mathrm{Gr}_{x}^{1 / 2}=G_{x}^{-1 / 2}$, where the Reynolds number $\left(\operatorname{Re}_{x}\right)$ is defined with $u_{\infty}$ as $u_{\infty} x / v$. While this rules out the possibility of having a self-similar solution, it also suggests that the following perturbation series for the non-dimensional velocity and temperature fields as,

$$
\begin{aligned}
& F=F_{0}(\eta)+\varepsilon F_{1}(\eta), \\
& H=H_{0}(\eta)+\varepsilon H_{1}(\eta),
\end{aligned}
$$

where the small parameter is defined as $\varepsilon=\operatorname{Re}_{x} / \mathrm{Gr}_{x}^{1 / 2}=G_{x}^{-1 / 2}$. 
For the solution of (7) and (8), the other required boundary conditions are given as:

$$
\begin{aligned}
& \text { at } y=0: u=0, \text { i.e., at } \eta=0: F^{\prime}=0, \\
& \text { at } y=0: v=0 \text {, i.e., at } \eta=0: F=0, \\
& \text { at } y=0: T=T_{W} \text {, i.e., at } \eta=0: H=1, \\
& \text { for large } y: u \rightarrow u_{\infty}, \text { i.e., for large } \eta: F \rightarrow G_{x}^{-1 / 2} \\
& \text { for large } y: T \rightarrow T_{\infty}, \text { i.e., for large } \eta: H \rightarrow 0 .
\end{aligned}
$$

For the perturbation expansion of (9) and (10), $F_{0}$ and $H_{0}$ are the components that apply to purely free convection problem. Substituting these into (7) and ignoring $o\left(\varepsilon^{2}\right)$ terms we get:

$$
F_{0}^{\prime \prime \prime}+\varepsilon F_{1}^{\prime \prime \prime}+\frac{3}{4} F_{0} F_{0}^{\prime \prime}+\varepsilon \frac{3}{4} F_{0} F_{1}^{\prime \prime}+\varepsilon \frac{3}{4} F_{1} F_{0}^{\prime \prime}-\frac{F_{0}^{\prime 2}}{2}-\varepsilon F_{0}^{\prime} F_{1}^{\prime}+H_{0}+\varepsilon H_{1}=0 .
$$

Thus, it follows for free convection that the following governing equation defines the selfsimilar solution,

$$
F_{0}^{\prime \prime \prime}+(3 / 4) F_{0} F_{0}^{\prime \prime}-\left(F_{0}^{\prime 2} / 2\right)+H_{0}=0 .
$$

Departure from the self-similar solution is given by the solution of the following,

$$
F_{1}^{\prime \prime \prime}+(3 / 4) F_{0} F^{\prime \prime}+(3 / 4) F_{1} F_{0}^{\prime \prime}-F_{0}^{\prime} F_{1}^{\prime}+H_{1}=0 .
$$

Boundary conditions for (11) and (12) are given by,

$$
\begin{aligned}
& \eta=0, F_{0}^{\prime}=0, F_{1}^{\prime}=0, \\
& \eta=0, F_{0}=0, F_{1}=0, \\
& \eta=0, H_{0}=1, H_{1}=0 .
\end{aligned}
$$

For very large

$$
\eta: F_{0}^{\prime} \rightarrow 0, F_{1}^{\prime} \rightarrow 1,
$$

and:

$$
H_{0} \rightarrow 0, H_{1} \rightarrow 0 .
$$

Boundary condition on $F^{\prime}$, at large $\eta$, is obtained by noting that for large $\eta: F_{0}^{\prime}+\varepsilon F_{1}^{\prime} \rightarrow \varepsilon$. The free convective flow solution however requires that $F_{0}^{\prime} \rightarrow 0$ at large $\eta$, so the above equation requires: $F_{1}^{\prime} \rightarrow 1$

Similarly, (9) and (10) are substituted in (8) to yield up to first-order accuracy in $\varepsilon$ as,

$$
H_{0}^{\prime \prime}+\varepsilon H_{1}^{\prime \prime}+\frac{3}{4} \operatorname{Pr} F_{0} H_{1}^{\prime}+\frac{3}{4} \varepsilon \operatorname{Pr} F_{0} H_{1}^{\prime}+\frac{3}{4} \varepsilon \operatorname{Pr} F_{1} H_{0}^{\prime}=0 .
$$

Thus, the free convective flow solution is obtained from,

$$
H_{0}^{\prime \prime}+(3 / 4) \operatorname{Pr} F_{0} H_{0}^{\prime}=0,
$$

and the perturbation component is obtained from the solution of,

$$
H_{1}^{\prime \prime}+(3 / 4) \operatorname{Pr} F_{0} H_{1}^{\prime}+(3 / 4) \operatorname{Pr} F_{1} H_{0}^{\prime}=0 .
$$




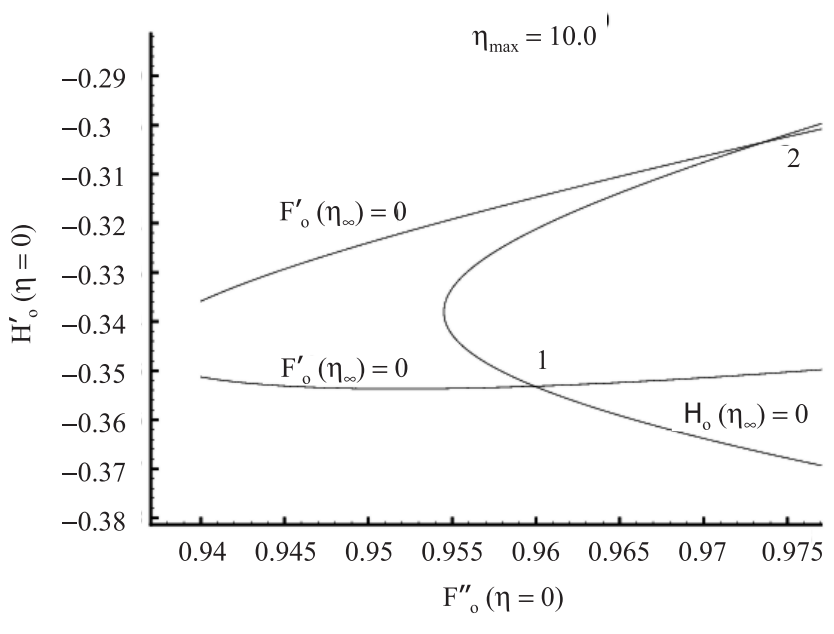

Figure 2. Grid search method values for $F_{0}^{\prime \prime}(0)$ and $H_{0}^{\prime}(0)$ to solve (11) and (13) using the shooting technique.

\section{Non-unique solution for assisting free convection flows}

The free convection problem is now defined in terms of the solution of (11) and (13) for the velocity and temperature fields respectively. Such solutions were obtained in Cebeci \& Bradshaw (1984) by using the Keller box method. This is essentially a matrix method that solves the discretized equations applied at all the nodes simultaneously, satisfying the boundary conditions as given above for $F_{0}$ and $H_{0}$.

From the numerical solution (as reported in Cebeci \& Bradshaw 1984), the dimensionless shear stress and heat transfer values at the surface of the plate are: $F_{0}^{\prime \prime}(\eta=0)=0.95578$ and $H_{0}^{\prime}(\eta=0)=-0 \cdot 35674$, for $\operatorname{Pr}=1$. Other values for wall shear stress and heat transfer rates are also tabulated for different values of Prandtl numbers in the same reference.

There is also an alternative method for solving (11) to (14) by the shooting technique (see Oosthuizen \& Naylor 1999), in which the problem is converted to an initial value problem by guessing the wall shear stress and heat transfer rates and iteratively improving the guess by the Newton-Raphson procedure. For the success of the latter procedure, we require very good initial guesses. To avoid this problem, we adopt instead the grid-search method, where we compute initial guesses at the nodes of the rectangular region discretized uniformly in the ranges: $0.94 \leq F_{0}^{\prime \prime}(\eta=0) \leq 0.99$ and $-0.38 \leq H_{0}^{\prime}(\eta=0) \leq-0.28$ with $(400 \times 1000)$ points, for the case of $\operatorname{Pr}=0 \cdot 7$. All calculations are done here using double precision and the error criterion in the shooting method converges to less than $10^{-10}$ with 8000 points in the $\eta$-direction for $0(1)$ equations. For $0(\varepsilon)$ equations, there are 4000 points in the $\eta$-direction. In figure 2 , two contour lines are identified along which the far-field boundary conditions on $F_{0}^{\prime}\left(\eta_{\infty}\right)$ and $H_{0}\left(\eta_{\infty}\right)$ are satisfied separately while solving (11) and (13). Therefore, in this figure the points 1 and 2 indicate combinations of $F_{0}^{\prime \prime}(0)$ and $H_{0}^{\prime}(0)$ for which the far-field conditions on $F_{0}^{\prime}\left(\eta_{\infty}\right)$ and $H_{0}\left(\eta_{\infty}\right)$ are simultaneously satisfied and these values can be used while solving (11) and (13) as an initial value problem. We refine the above values of wall shear and heat transfer rates using the Newton-Raphson search procedure, before actually using them for solving (11) to (14).

Equations (11) and (13) are solved as initial value problems by using the four-stage Runge-Kutta method from the wall up to $\eta=\eta_{\max }$, where the far-field boundary conditions 
are checked. Typical results are shown in figure 2 , for the choice of $\eta_{\max }=10$, by drawing the contours along which the far-field boundary conditions: $F_{0}^{\prime}\left(\eta=\eta_{\max }\right)=0$ and $H_{0}\left(\eta=\eta_{\max }\right)=0$ are satisfied separately. It can be shown that there are only two contour lines in the region of interest. Intersection of these two lines occurs at only two points as marked in the figure for which: $\left[F_{0}^{\prime \prime}(\eta=0)^{(1)}=0.959917049, H_{0}^{\prime}(\eta=0)^{(1)}=-0.353183959\right]$ and $\left[F_{0}^{\prime \prime}(\eta=0)^{(2)}=0.973500712, H_{0}^{\prime}(\eta=0)^{(2)}=-0.303573182\right]$ in the shown range for which (11) and (13) provide two self-similar solutions for free convection $(\varepsilon=0)$. To our knowledge, for vertical plates, such results of multiple solutions have not been reported before. However, for horizontal plates Schneider (1979) has shown that for a plate temperature distribution of the form $T_{w}=k x^{-0 \cdot 5}$, for $k \geq k_{0}<0$, a unique solution exists for a heated plate $(k \geq 0)$, while for a cooled plate $\left(k_{0}<k<0\right)$ two self-similar solutions exist. For different choices of $\eta=\eta_{\max }$ in the present exercise, a pair of such values has always been obtained.

Results obtained from the grid- search method are used as initial guesses for the NewtonRaphson method in the shooting technique. For all the cases investigated here for different $\eta_{\max }$, we have tabulated the wall values of $F_{o}^{\prime \prime}(0), F_{1}^{\prime \prime}(0), H_{0}^{\prime}(0)$ and $H_{1}^{\prime}(0)$ in table 1 . In this table, plausible wall values of shear stress and heat transfer rates have been reported for

Table 1. Wall boundary conditions for (11) to (14) for different lengths of the domain ( $\operatorname{Pr}=0 \cdot 7)$.

\begin{tabular}{|c|c|c|c|c|c|}
\hline$\eta_{\max }$ & Solution & $F_{0}^{\prime \prime}(0)$ & $H_{0}^{\prime}(0)$ & $F_{1}^{\prime \prime}(0)$ & $H_{1}^{\prime}(0)$ \\
\hline \multirow[t]{2}{*}{$7 \cdot 0$} & 1 & 0.957133366 & -0.35342305 & $0 \cdot 045321213$ & $-0 \cdot 046027359$ \\
\hline & 2 & 0.973659973 & $-0 \cdot 20078645$ & 0.021917066 & 0.0416641735 \\
\hline \multirow[t]{2}{*}{$8 \cdot 0$} & 1 & 0.958912741 & $-0 \cdot 353214913$ & 0.03788334 & -0.044968878 \\
\hline & 2 & 0.981593498 & $-0 \cdot 251083577$ & $0 \cdot 033831512$ & $0 \cdot 0476564158$ \\
\hline \multirow[t]{2}{*}{$9 \cdot 0$} & 1 & 0.959626375 & $-0 \cdot 353179568$ & 0.036765896 & $-0 \cdot 044165686$ \\
\hline & 2 & 0.978468418 & $-0 \cdot 283205262$ & $0 \cdot 040213285$ & $0 \cdot 0493605548$ \\
\hline \multirow[t]{2}{*}{$10 \cdot 0$} & 1 & 0.959917049 & $-0 \cdot 353183959$ & 0.031304859 & $-0 \cdot 0436021452$ \\
\hline & 2 & 0.973500730 & $-0 \cdot 303573162$ & $0 \cdot 043822799$ & 0.04897131347 \\
\hline \multirow[t]{2}{*}{$10 \cdot 05$} & 1 & 0.959925933 & $-0 \cdot 353184440$ & 0.031218684 & -0.0435793147 \\
\hline & 2 & 0.9732593180 & $-0 \cdot 3043720230$ & 0.0439375046 & 0.0489209246 \\
\hline \multirow[t]{2}{*}{$10 \cdot 06$} & 1 & 0.959927634 & $-0 \cdot 353184512$ & $0 \cdot 031201725$ & -0.043574917 \\
\hline & 2 & 0.973211345 & -0.304529703 & 0.0439596353 & $0 \cdot 048910330$ \\
\hline \multirow[t]{2}{*}{$10 \cdot 07$} & 1 & 0.959929361 & $-0 \cdot 353183429$ & - & - \\
\hline & 2 & 0.973163355 & $-0 \cdot 304686645$ & 0.0439818087 & 0.04890019269 \\
\hline \multirow[t]{2}{*}{$10 \cdot 073$} & 1 & 0.959930053 & $-0 \cdot 353184585$ & - & - \\
\hline & 2 & 0.973148973 & -0.304733546 & $0 \cdot 0439882583$ & $0 \cdot 04889680566$ \\
\hline \multirow[t]{2}{*}{$10 \cdot 50$} & 1 & 0.959990263 & -0.353188671 & - & - \\
\hline & 2 & 0.971180556 & -0.310828785 & $0 \cdot 0447314874$ & $0 \cdot 0483844416$ \\
\hline \multirow[t]{2}{*}{$11 \cdot 0$} & 1 & 0.960037497 & $-0 \cdot 353193080$ & - & - \\
\hline & 2 & 0.969100784 & $-0 \cdot 316683330$ & $0 \cdot 0451570320$ & $0 \cdot 0476581477$ \\
\hline
\end{tabular}




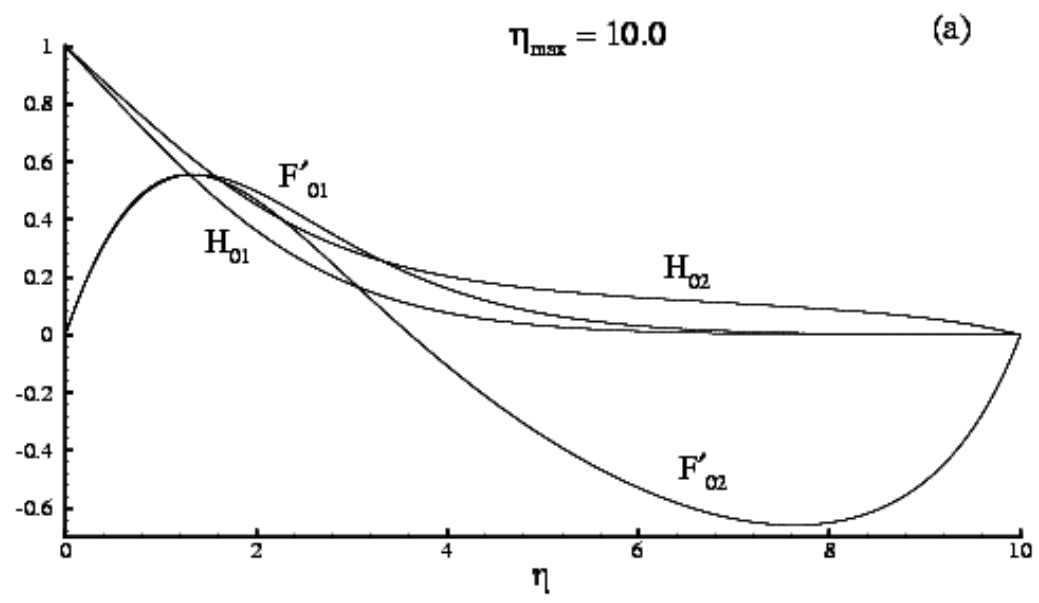

(b)

(c)
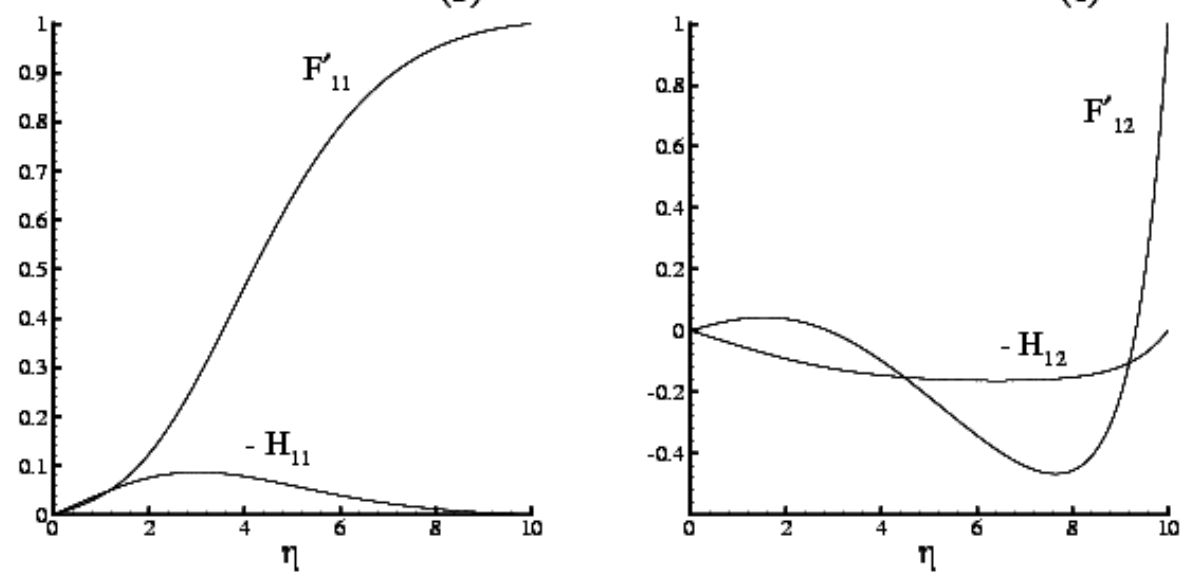

Figure 3. (a) Similarity solutions of (11) and (13) for $\eta_{\max }=10$ obtained by shooting method, using wall conditions obtained from figure 2 as initial guesses. (b) \& (c) The perturbative solution of (12) and (14) corresponding to $0(1)$ solutions of figure $3 \mathrm{a}$.

different choices of $\eta=\eta_{\max }$. The wall values are provided for both the self-similar solution $(\varepsilon=0)$ and its perturbation - the $0(\varepsilon)$ solution. Thus, this exercise shows the existence of two solutions for all free convection cases with different $\eta_{\max }$. In figure $3 \mathrm{a}$, these two selfsimilar solutions are shown for $\eta_{\max }=10$ having the following nature. The first solution shows the velocity $\left(F_{01}^{\prime}\right)$ to be positive everywhere in the investigated domain, while the nondimensional temperature $\left(H_{01}\right)$ drops monotonically with height starting from the plate. The second solution also shows the temperature $\left(H_{02}\right)$ to drop monotonically with height, but the velocity field $\left(F_{02}^{\prime}\right)$ shows the flow to be recirculating in the outer part of the domain. It is for this attribute, we term the second solution as non-physical - one which has not been recorded experimentally. The first solution is noted experimentally and reported in the literature, as in Brewster \& Gebhart (1991). Both the self-similar solutions indicate correct heat transfer from the plate to the surrounding fluid - as expected for assisting flows. 


\section{Breakdown of asymptotic expansion}

Of the pair of self-similar solutions obtained in the previous section, not all admit valid perturbation expansion given by (9) and (10). This is explained next. Having obtained the values of $F_{0}$ and $H_{0}$, we use these in (12) and (14) to solve for $F_{1}(\eta)$ and $H_{1}(\eta)$ using the "shooting technique". In figures $3 \mathrm{~b}$ and $\mathrm{c}$ the perturbative component of these two solutions are shown. The perturbed velocity field shows the desired monotonic growth to its free stream value in figure $3 \mathrm{~b}$, as given by the solution of (12). The perturbed temperature field is as shown in Brewster \& Gebhart (1991). The second perturbed solution shown in 3c indicates that the velocity field is mostly in the reverse direction except near the upper edge of the domain. Also, the perturbed temperature field is of opposite type to that shown in figure $3 \mathrm{~b}$. Brewster \& Gebhart (1991) did not identify this solution or report its mathematical existence.

In figure 4 , the similarity solutions and its perturbation components are shown for the case where $\eta_{\max }=7$, obtained by solving (11) to (14) in the prescribed domain. This domain

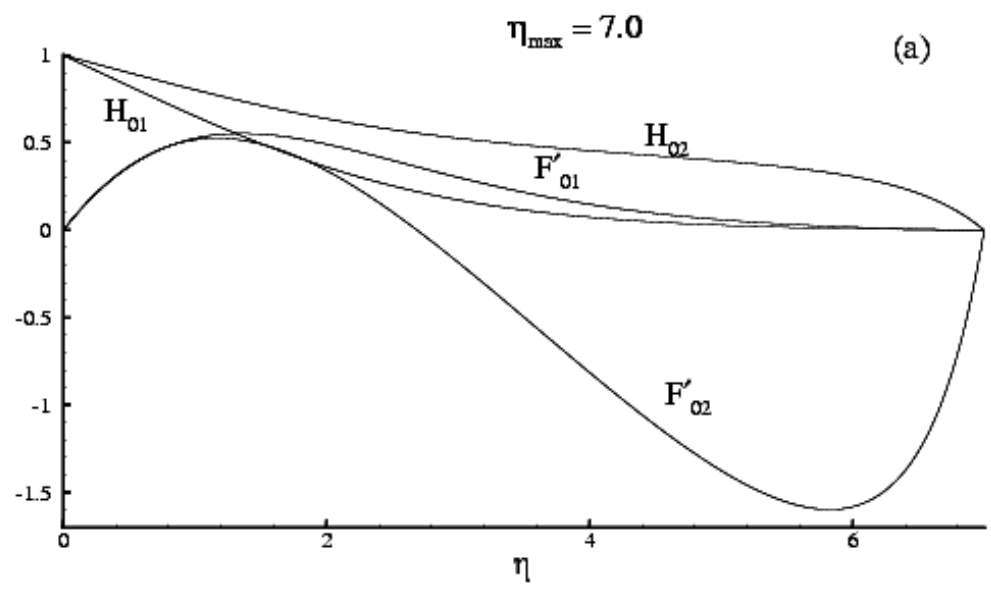

(b)

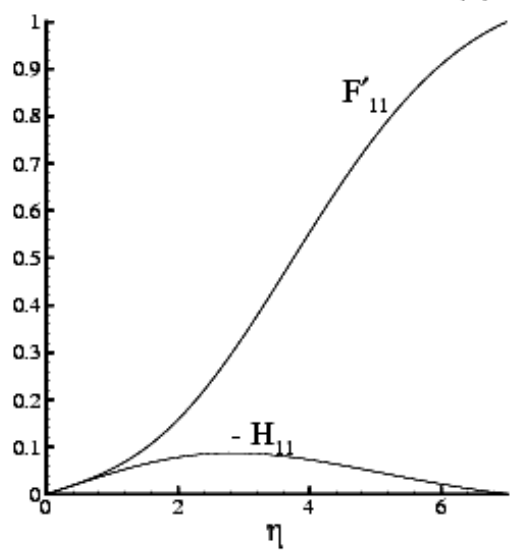

(c)

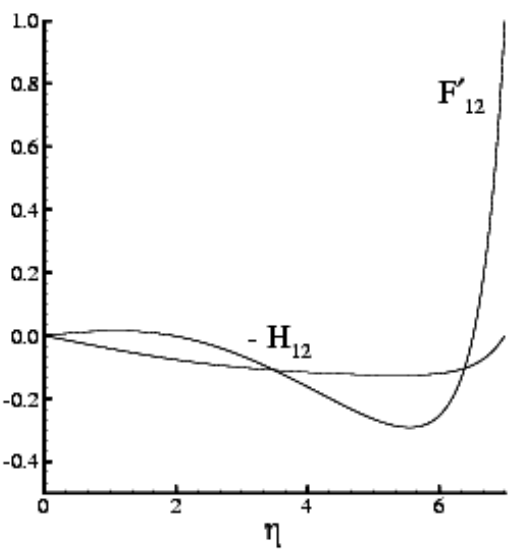

Figure 4. (a) Similarity solutions of (11) and (13) for $\eta_{\max }$ obtained by shooting method, using wall conditions obtained from table 1 as initial guesses. (b) \& (c) The perturbative solution of (12) and (14) corresponding to $0(1)$ solution of figure $4 \mathrm{a}$. 


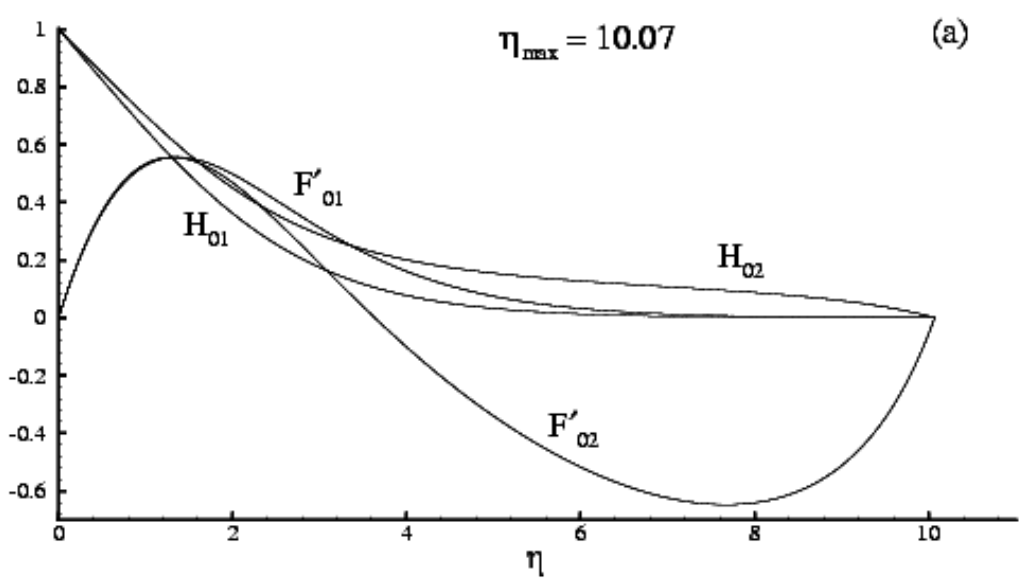

(b)

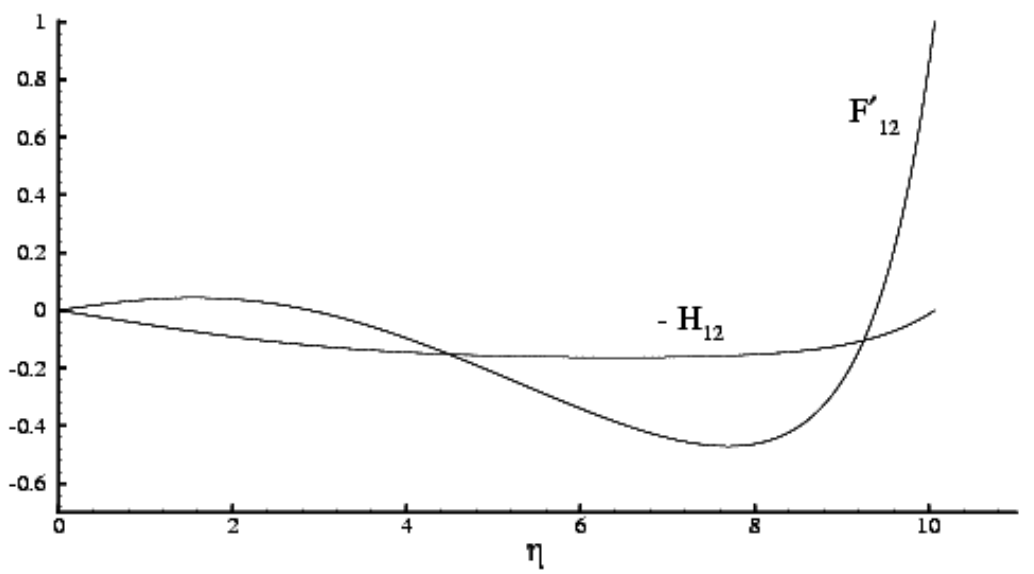

Figure 5. (a) Similarity solutions of (11) and (13) for $\eta_{\max }=10.07$ obtained by shooting method, using wall conditions obtained from table 1 as initial guesses. (b) The perturbative solution of (12) and (14) corresponding to $0(1)$ solution of ' 2 ' in figure 5 a.

was also used in Cebeci \& Bradshaw (1984) and Brewster \& Gebhart (1991). Therefore, the present results can be compared with these solutions. The qualitative nature of the solutions remains the same, as that was found for $\eta_{\max }=10$ and shown in figure 3. In fact, for any values of $\eta_{\max }$ between 7 and 10.06, the qualitative nature of the solution remains the same. Furthermore, the solutions for $F_{01}^{\prime}, H_{01}$ are the same that is reported in Cebeci \& Bradshaw (1984) and Brewster \& Gebhart (1991). The perturbative solution shown in figure 4b also matches with the solution given in Brewster \& Gebhart (1991).

When $\eta_{\max }$ is increased to 10.07 , both the similarity solutions are shown in figure $5 \mathrm{a}$, having similar properties as discussed above. However, the perturbative components $F_{11}^{\prime}$ and $H_{11}$ did not converge following the shooting technique, when a solution was sought for (12) and (14) using the $0(1)$ solution given by (11) and (13). The other solution, given by $F_{12}^{\prime}$ and $H_{12}$, provided a converged solution and shown in figure $5 \mathrm{~b}$, similar to the solution obtained before for lower $\eta_{\max }$. Thus, the perturbed solution exists only for the non-physical similarity 


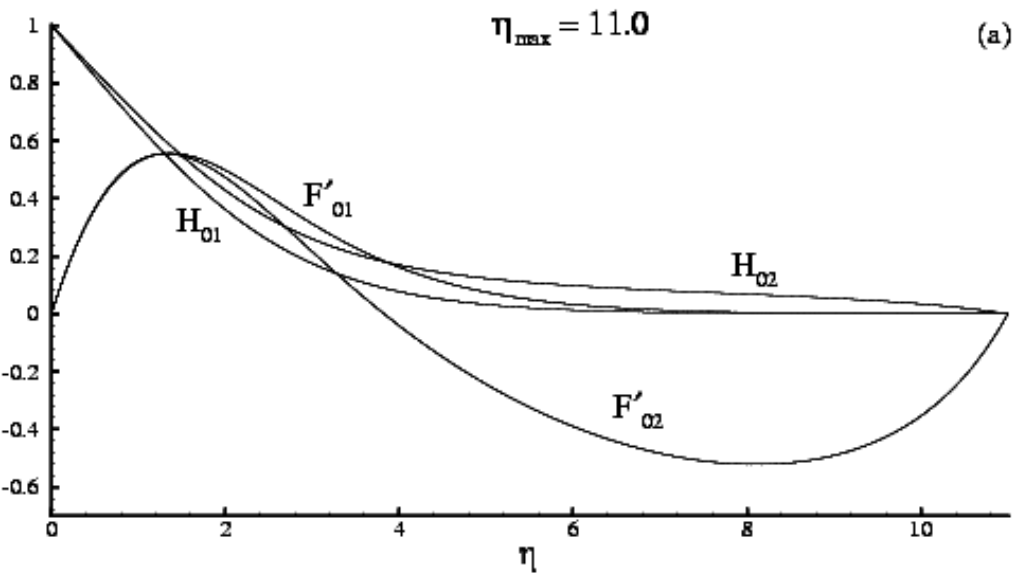

(a)

(b)

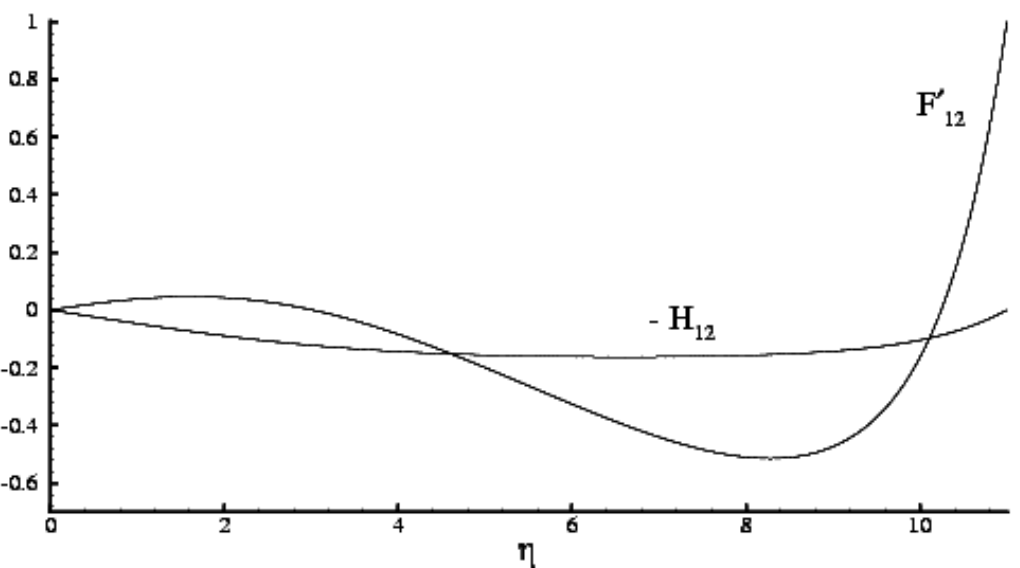

Figure 6. (a) Similarity solutions of (11) and (13) for $\eta_{\max }=11$ obtained by shooting method, using wall conditions obtained from table 1 as initial guesses. (b) The perturbative solution of (12) and (14) corresponding to $0(1)$ solution of ' 2 ' in figure 6 a.

solution, indicating a breakdown of the perturbation process for $\eta_{\max }$ greater than $10 \cdot 06$. This type of breakdown of asymptotic solution for one set of $0(1)$ solution continued for higher values of $\eta_{\max }$. In figures $6 \mathrm{a}$ and $\mathrm{b}$ the corresponding $0(1)$ and the converged $0(\varepsilon)$ solutions are shown for $\eta_{\max }$ equal to 11 .

\section{Conclusions}

Non-unique, non-convergent solutions (by the shooting technique), are reported here for the first time, for non-similar assisting flow past vertical flat plates. The non-similar solution is obtained in terms of perturbation series, whose $0(1)$ solution is nothing but the solution for natural convection past a vertical plate. The perturbed solution is obtained in terms of the small parameter, $\varepsilon=G_{x}^{-0 \cdot 5}$. It is found that the $0(1)$ part has two solutions, one that is 
indicated in the literature having the proper monotonically growing feature, as in Cebeci \& Bradshaw (1984) and Brewster \& Gebhart (1991). The second solution is not physical, as the flow exhibits recirculation in the outer part of the boundary layer. This is true for any choice of integration domain for the wall-normal distance $\left(\eta_{\max }\right)$.

However, $O(\varepsilon)$ solution for the above two branches of $0(1)$ solution may or may not exist, depending upon the choice of $\eta_{\max }$. For $\eta_{\max } \leq 10 \cdot 06$, we have two $0(\varepsilon)$ solutions. For $\eta_{\max }$ greater than this value, only one solution exists, corresponding to the non-physical 0(1) solution. Thus, we see that $\eta_{\max }$ is the limiting factor in obtaining a proper physical solution for this case of assisting flow past vertical flat plates.

\section{References}

Brewster R A, Gebhart B 1991 Instability and disturbance amplification in a mixed-convection boundary layer. J. Fluid Mech. 229: 115-133

Cebeci T, Bradshaw P 1984 Physical and computational aspects of convective heat transfer (New York: Springer-Verlag)

Oosthuizen P H, Naylor D 1999 An introduction to convective heat transfer analysis (Singapore: WCB/McGraw-Hill)

Schneider W 1979 A similarity solution for combined forced and free convection flow over a horizontal plate. Int. J. Heat Transfer 22: 1401-1406

Schneider W, Wasel M G 1985 Breakdown of the boundary-layer approximation for mixed convection above a horizontal plate. Int. J. Heat Transfer 28: 2307-2313

Steinrück H 1994 Mixed convection over a cooled horizontal plate: non-uniqueness and numerical instabilities of the boundary-layer equations. J. Fluid Mech. 278: 251-265 